\title{
Selenocysteine-mediated native chemical ligation
}

\section{Doctoral Thesis}

Author(s):

Quaderer, Richard Johann

Publication date:

2002

Permanent link:

https://doi.org/10.3929/ethz-a-004378163

Rights / license:

In Copyright - Non-Commercial Use Permitted 
Diss. ETH No. 14555

\title{
Selenocysteine-Mediated Native Chemical Ligation
}

\author{
A dissertation submitted to the \\ Presented by \\ Richard Johann Quaderer \\ Dipl. Chem. ETH \\ Born July 10, 1972 \\ From Liechtenstein
}

Swiss Federal Institute of Technology (ETH) Zurich

Accepted on the recommendation of

Prof. Dr. Donald Hilvert, examiner

Prof. Dr. Pier Luigi Luisi, co-examiner

Zurich, 2002 


\section{Abstract}

In this thesis selenocysteine-mediated native chemical ligation of unprotected peptide fragments was established. This new condensation method for the production of selenopeptides and -proteins was applied to the total chemical synthesis of a selenocysteine-containing variant of bovine pancreatic trypsin inhibitor (BPTI). Furthermore, a second diselenide-containing variant of BPTI was produced by chemical synthesis to study the disulfide-coupled folding pathway of BPTI. In addition, selenocysteine was shown to effect peptide cyclization and was converted to natural and non-natural amino acids after cyclization in order to expand the scope of this new method.

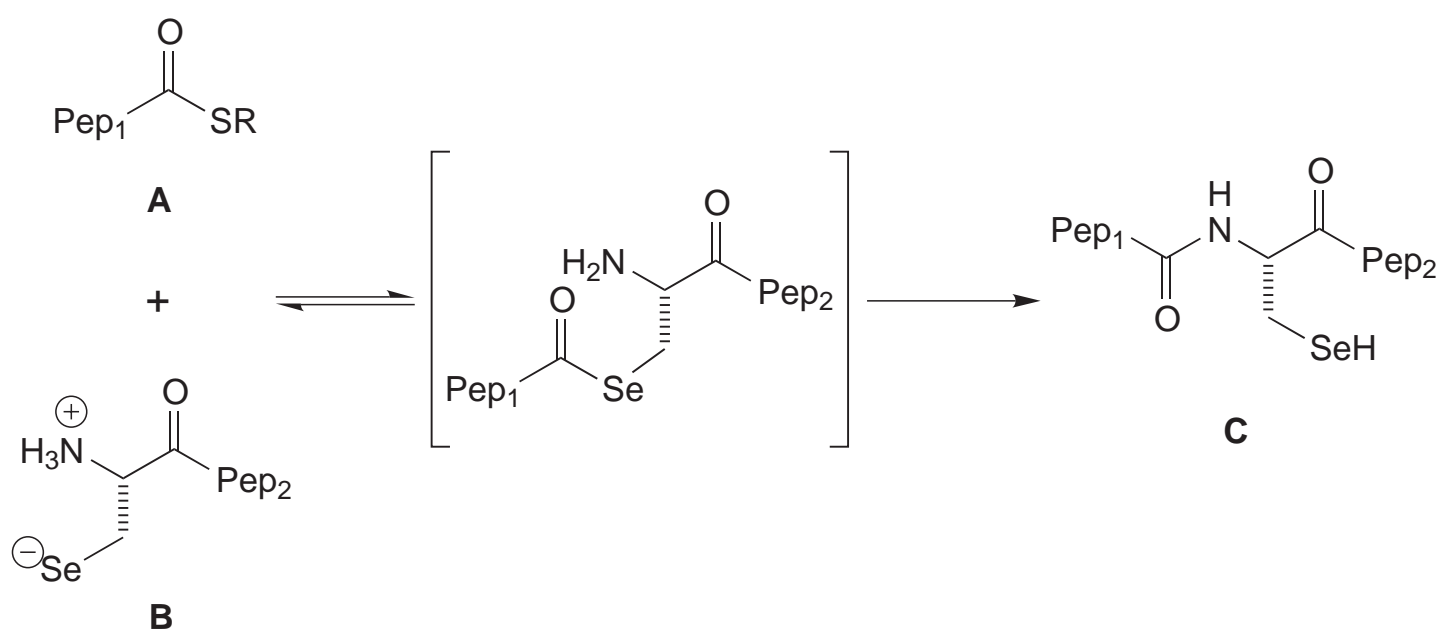

Scheme 1: Selenocysteine-mediated native chemical ligation. Conditions: Buffer (100 mM phosphate $\mathrm{pH}$ 6-9), $6 \mathrm{M} \mathrm{GdmCl}$, PhSH 3\% (v/v).

C-Terminal peptide thioesters $\mathbf{A}$ were shown to react efficiently with peptide fragments containing an $\mathrm{N}$-terminal selenocysteine $\mathbf{B}$ to give selenopeptides and selenoproteins $\mathbf{C}$ (Scheme 1). In analogy to the native chemical ligation of thioesters and peptides containing $\mathrm{N}$-terminal cysteines, the selenol presumably attacks the thioester nucleophilically to give a selenoester intermediate that subsequently rearranges to give a native amide bond.

Suitable reaction conditions were established by model ligations of the pentapeptide thioethyl ester LYRAG-SEt with selenocystine, the diselenide dimer of selenocysteine. It was found that reaction is effected by simply 
dissolving both fragments in aqueous buffer in a $\mathrm{pH}$ range of ca. 6 to 9 (containing $6 \mathrm{M}$ guanidinium chloride, $\mathrm{GdmCl}$, to increase solubility). Addition of thiophenol $(3 \%, v / v)$ not only increases reactivity of the thioester moiety through transthioesterification, as previously reported, but additionally serves as reducing agent for generation of the nucleophilic selenol in situ.

The utility of this procedure was demonstrated by the synthesis of a selenium-containing derivative of BPTI in which Cys38 is replaced by selenocysteine. The full-length peptide was obtained by ligation of the $\mathrm{N}$ terminal fragment BPTI(1-37)-SEt thioester with Cys38Sec-BPTI(38-58). This artificial selenoprotein was shown to fold into a conformation similar to that of wild-type BPTI and to inhibit trypsin and chymotrypsin with unaltered affinity.

In the second part of this thesis, a second variant of BPTI containing two selenocysteines, Cys5Sec/Cys55Sec-BPTI(2-58), was produced. In this protein, the disulfide bridge between positions 5 and 55 is replaced by a diselenide. This variant was synthesized to investigate the highly controversial disulfide-coupled folding pathway of BPTI. It was obtained via condensation of fragments Cys5Sec-BPTI(2-37)- $\mathrm{SCH}_{2} \mathrm{CH}_{2} \mathrm{CO}_{2}$ Et thioester and Cys55Sec-BPTI(38-58) by cysteine-mediated native chemical ligation. Since the protected selenocysteinederivative suitable for Fmoc-based solid-phase peptide synthesis is basesensitive, special coupling protocols had to be used to obtain the fragments. In addition, during the synthesis of the $\mathrm{N}$-terminal thioester fragment of Cys5Sec/Cys55Sec-BPTI(2-37) we encountered problems (very low yields) with the alkanesulfonamide "safety-catch" linker (Scheme 2). The yield of isolated thioester $\mathbf{A}$ could be increased significantly when the cleavage reaction of peptide-resin $\mathbf{C}$ was carried out in $2 \mathrm{M} \mathrm{LiBr/THF}$ rather than DMF or THF. The largest effects were seen with problematic peptides that aggregate or form secondary structures on the resin. This finding might prove useful for other applications, since others have encountered similar problems. 


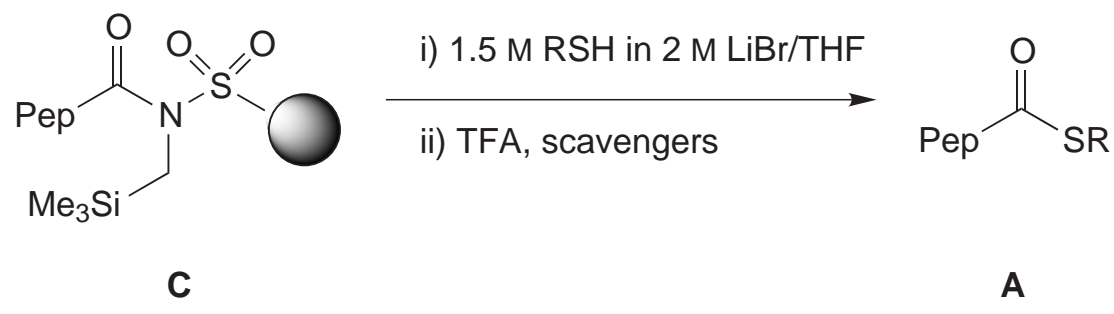

Scheme 2: "Safety-catch" linker for C-terminal peptide thioester synthesis. Use of $2 \mathrm{M} \mathrm{LiBr} / \mathrm{THF}$ for the cleavage reaction of peptide-resins $\mathbf{C}$ significantly increased yields of $C$-terminal thioester peptides $\mathbf{A}$.

The final protein, Cys5Sec/Cys55Sec-BPTI(2-58), was shown to fold into a native-like structure that is fully biologically active as judged by inhibition of trypsin and chymotrypsin. Although further experiments are necessary, this preliminary finding indicates that the folding pathway of BPTI suggested by Creighton is not fully correct. Creighton's folding scheme postulates the necessity of non-native disulfide intermediates to adopt the fully oxidized native fold. Because of the presence of a diselenide bridge between positions 5 and 55 in Cys5Sec/Cys55Sec-BPTI(2-58), this variant is unable to form those nonnative disulfides but still establishes the native fold.

In the last part of this work selenocysteine-mediated native chemical ligation was shown to be useful for the preparation of cyclic peptides. A linear 16meric peptide featuring an $\mathrm{N}$-terminal selenocysteine and a $\mathrm{C}$-terminal thioester was cyclized by simply dissolving it in $100 \mathrm{mM}$ phosphate buffer ( $\mathrm{pH} \mathrm{7.5)}$ containing $3 \%$ thiophenol $(\mathrm{v} / \mathrm{v})$. After cyclization, selenocysteine was converted to three other amino acids. First, reaction with iodoacetamide served as model to demonstrate that a variety of electrophiles can be attached to the selenol side chain in peptides. Second, selenocysteine was converted to alanine, a proteinogenic amino acid, by metal-catalyzed reduction. Together with the recently reported conversion of cysteine to alanine, this finding significantly widens the scope of native chemical ligation. Third, dehydroalanine was obtained from selenocysteine by $\beta$-elimination. The resulting Michael acceptor reacts readily with nucleophiles. The cyclic dehydroalanine-containing model peptide rapidly underwent an intramolecular reaction by attack of a nucleophilic residue. 
In conclusion, a novel method to obtain both natural and unnatural selenoproteins has been developed. Modification of the selenol side chain after ligation further broadens the scope of selenocysteine-mediated native chemical ligation. This method is thus likely to be useful in the more general context of methods to join peptides at a wider variety of sites than has been possible heretofore and to generate tailored side chains exhibiting specific properties after ligation. 


\section{Zusammenfassung}

In dieser Dissertation wurde die Selenocystein-vermittelte Native-ChemicalLigation von ungeschützten Peptidfragmenten erarbeitet. Diese neue Kondensationsmethode zur Herstellung von Selenopeptiden und -proteinen wurde auf die chemische Totalsynthese einer Selenocystein-enthaltenden Mutante von Bovine-Pancreatic-Trypsin-Inhibitor (BPTI) angewendet. Eine zweite Mutante von BPTI, welche eine Diselenidbrücke enthält, wurde ebenfalls durch chemische Totalsynthese hergestellt, um den Faltungsweg von BPTI zu untersuchen, welcher an die Bildung von Disulfid-Brücken gekoppelt ist. Zusätzlich wurde gezeigt, dass Selenocystein zur Peptidzyklisierung benutzt werden kann. Nach der Zyklisierung wurde Selenocystein in natürliche und unnatürliche Aminosäuren umgewandelt, um den Anwendungsbereich dieser neuen Methode zu erweitern.

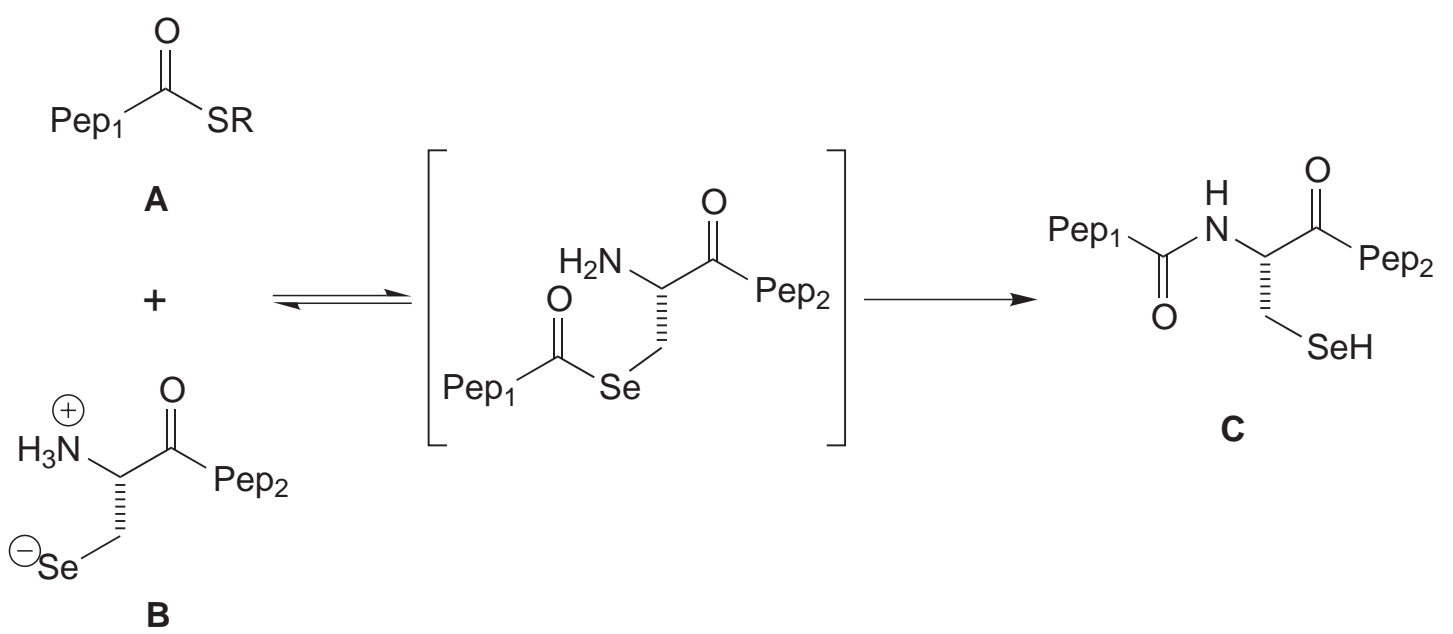

Schema 1: Selenocystein-vermittelte Native-Chemical-Ligation. Reaktionsbedingungen: 100 mM Phosphat-Puffer pH 6-9, 6 M GdmCl, PhSH 3\% (v/v).

Es wurde gezeigt, dass sich $C$-terminale Peptidthioester A mit Peptidfragmenten B, welche ein $\mathrm{N}$-terminales Selenocystein enthalten, gut zu Selenopeptiden und Selenoproteinen $\mathbf{C}$ umsetzen lassen (Schema 1). Analog zur Native-Chemical-Ligation von Thioestern und Peptiden mit einem $\mathrm{N}$ terminalen Cystein greift das Selenol vermutlich den Thioester nukleophil an, 
um ein Selenoester-Intermediat zu bilden, welches rasch unter Bildung einer natürlichen Amidbindung umlagert.

Geeignete Reaktionsbedingungen wurden anhand von Modelligationen des Pentapeptidthioethylesters LYRAG-SEt mit Selenocystin, dem Diselenid-Dimer von Selenocystein, erarbeitet. Es wurde gefunden, dass die Reaktion durch einfaches Auflösen der beiden Fragmente in wässrigem Puffer in einem $\mathrm{pH}$ Bereich von ca. 6 bis 9 unter Zusatz von $6 \mathrm{M}$ Guanidiniumchlorid (GdmCl). Die Zugabe von Thiophenol $(3 \%, v / v)$ führt zu einer Erhöhung der Reaktivität des Thioesters durch Thiolaustausch, und dient als Reduktionsmittel, um das nukleophile Selenol in situ zu generieren.

Die Nützlichkeit der Native-Chemical-Ligation wurde durch die Synthese einer Variante von BPTI demonstriert, in welcher Cys38 durch Selenocystein substitutiert ist. Das Polypeptid wurde durch Ligation des $\mathrm{N}$-terminalen Thioesterfragmentes BPTI(1-37)-SEt mit Cys38Sec-BPTI(38-58) erhalten. Es wurde gezeigt, dass sich dieses Selenoprotein in eine dem Wildtyp-BPTI vergleichbare Konformation faltet und sowohl Trypsin als auch Chymotrypsin mit unveränderter Affinität hemmt.

Im zweiten Teil dieser Arbeit wurde eine zweite Variante von BPTI, die zwei Selenocysteinreste enthält, Cys5Sec/Cys55Sec-BPTI(2-58), hergestellt. In diesem Protein ist die Disulfidbrücke zwischen den Positionen 5 und 55 durch ein Diselenid ersetzt. Diese Mutante wurde synthetisiert, um den umstrittenen Disulfid-gekoppelten Faltungsweg von BPTI zu untersuchen. Erhalten wurde dieses Protein durch Kondensation der Fragmente Cys5Sec-BPTI(2-37)$\mathrm{SCH}_{2} \mathrm{CH}_{2} \mathrm{CO}_{2}$ Et und Cys55Sec-BPTI(38-58) via Cystein-vermittelter NativeChemical-Ligation. Aufgrund der ausgeprägten Basenlabilität des für die Fmocbasierende Festphasenpeptidsynthese geeigneten Selenocysteinderivates, mussten spezielle Kupplungsprotokolle zur Synthese der Fragmente angewendet werden. Zusätzlich traten Schwierigkeiten (sehr niedrige Ausbeuten) während der Synthese des $\mathrm{N}$-terminalen Thioesterfragments von Cys5Sec/Cys55Sec-BPTI(2-37) mit dem Alkansulfonamid "safety-catch" Linker 
auf (Schema 2). Die Ausbeuten an Thioester A konnten deutlich gesteigert werden, wenn die Abspaltungsreaktion des Peptid-Harzes $\mathbf{C}$ in $2 \mathrm{M} \mathrm{LiBr} / \mathrm{THF}$ anstelle von reinem DMF oder THF durchgeführt wurden. Die grössten Steigerungen der Ausbeute traten mit problematischen Peptiden auf, welche auf dem Trägerharz Aggregate oder Sekundärstrukturen ausbilden. Diese Beobachtung könnte auch für andere Anwendungen nützlich sein, vor allem da ähnliche Probleme in der Literatur beschrieben wurden.

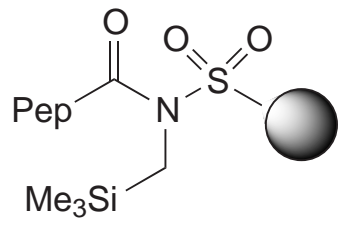

C i) $1.5 \mathrm{M} \mathrm{RSH}$ in $2 \mathrm{M} \mathrm{LiBr} / \mathrm{THF}$

ii) TFA, scavengers

A

Schema 2: Der "safety-catch" Linker für die Synthese von C-terminalen Peptidthioestern. Durch Einsatz von $2 \mathrm{M} \mathrm{LiBr/THF}$ als Lösungsmittel für die Spaltungsreaktion des Peptidharzes $\mathbf{C}$ konnten die Ausbeuten an $C$-terminalen Thioesterpeptiden $\mathbf{A}$ deutlich erhöht werden.

Das resultierende Protein, Cys5Sec/Cys55Sec-BPTI(2-58), faltet in eine dem Wildtyp-BPTI ähnliche Struktur mit unverminderter biologischer Aktivität. Dies konnte durch die Inhibierung von Trypsin und Chymotrypsin gezeigt werden. Obwohl weitere Experiments notwendig sind, deutet dieser vorläufige Befund darauf hin, dass der von Creighton vorgeschlagene Faltungsweg von BPTI nicht gänzlich korrekt ist. Das Faltungsschema von Creighton postuliert die Notwendigkeit von nicht-nativen Disulfid-Intermediaten um das voll oxidierte, nativ gefaltete Protein zu erhalten. Aufgrund der Präsenz einer Diselenidbrücke zwischen den Positionen 5 und 55 in Cys5Sec/Cys55Sec-BPTI(2-58), kann diese Mutante jene nicht-nativen Disulfide nicht formen. Dennoch faltet die Variante analog zum Wildtyp.

Im letzten Teil dieser Arbeit wurde gezeigt, dass die Selenocysteinvermittelte Native-Chemical-Ligation zur Synthese von zyklischen Peptiden eingesetzt werden kann. Ein lineares 16 meres Peptid, welches ein $N$-terminales Selenocystein und einen $C$-terminalen Thioester enthält, wurde durch Lösen in $3 \%$ Thiophenol $(\mathrm{v} / \mathrm{v})$ enthaltendem Phosphat-Puffer (100 mM, pH 7.5) zyklisiert. 
Selenocystein wurde nach der Zyklisierung in drei verschiedene Aminosäuren umgewandelt. Zuerst wurde mit lodoacetamid umgesetzt, um zu zeigen, dass unterschiedliche Elektrophile mit der Selenol-Seitenkette in Peptiden verknüpft werden können. Zweitens wurde Selenocystein durch metallkatalysierte Reduktion in die proteinogene Aminosäure Alanin umgewandelt. Gemeinsam mit der kürzlich beschriebenen Umwandlung von Cystein in Alanin erweitert dieses Ergebnis den Anwendungsbereich der Native-Chemical-Ligation beträchtlich. Drittens wurde Dehydroalanin via $\beta$-Elimination der SelenocysteinSeitenkette erhalten. Der resultierende Michael-Akzeptor reagiert leicht mit Nukleophilen. Das zyklische Dehydroalanin-enthaltende Modellpeptid reagiert durch intramolekularen Angriff einer nukleophilen Seitenkette schnell weiter.

Zusammenfassend wurde eine neue Methode zur Herstellung sowohl natürlicher als auch unnatürlicher Selenoproteine entwickelt. Die Modifizierung der Selenol-Seitenkette nach der Ligation erweitert den Anwendungsbereich der Selenocystein-vermittelten Native-Chemical-Ligation. Es ist anzunehmen, dass diese Methode nützlich sein wird im generelleren Kontext von Methoden, um Peptide an einer grösseren Bandbreite von Verknüpfungsstellen als es bisher möglich war, zu kondensieren, und um definierte Seitenketten mit spezifischen Eigenschaften nach der Ligation einzuführen. 